\title{
Tamaño relativo encefálico e índices cerebrales en Vanellus c. chilensis (Aves: Charadriidae)
}

\author{
Relative encephalic size and cerebral indices of Vanellus c. chilensis \\ (Aves: Charadriidae)
}

ESTELA PISTONE, FERNANDO CAREZZANO \& NOEMÍ BEE-DE-SPERONI

Cátedra de Anatomía Comparada, Departamento de Diversidad Biológica y Ecología, Escuela de Biología, Facultad de Ciencias Exactas, Físicas y Naturales, Universidad Nacional de Córdoba, Avenida Vélez Sarsfield 299, 5000 Córdoba, Argentina; e-mail: fercarezza@yahoo.com

\begin{abstract}
RESUMEN
Se analizó la composición cuantitativa encefálica y se estimaron índices cerebrales en Vanellus c. chilensis (tero o queltehue). Se estimó el volumen porcentual e índices cerebrales del encéfalo total y de siete de sus componentes, como así también los núcleos de relevo de las vías trigeminal, visual y acústica. El telencéfalo es el componente de mayor volumen relativo, siendo el neoestriado la estructura telencefálica de tamaño superior. El desarrollo del estriado propiamente dicho, tecto óptico y los núcleos de relevo de las vías visual y trigeminal concuerdan con la dieta carnívora de Vanellus c. chilensis. El tamaño relativo del Wulst y de los núcleos de la vía acústica se asocia a las complicadas tácticas que utiliza esta especie en la defensa del nido. Los índices cerebrales de las estructuras encefálicas analizadas indican que Vanellus c. chilensis es un ave progresiva.
\end{abstract}

Palabras clave: Vanellus c. chilensis, neuroanatomía, tamaño relativo, ecología, comportamiento.

\begin{abstract}
The quantitative encephalic composition and cerebral indices of Vanellus c. chilensis (southern lapwing ) were analyzed. The percentual volumes and cerebral indices for the whole encephalon and for seven components were calculated as well as relevous nuclei of the trigeminal, visual and acoustic pathways. The component of greater relative volume is the telencephalon. The neostriatum is the most developed encephalic structure. Developing of bulbus olfactorius, striatum, tectum opticum and relevous nuclei of visual and trigeminal pathways are according with the carnivorous diet of Vanellus c. chilensis. The relative size of Wulst and relevous nuclei of acoustic pathway appears associated with the complex tactics used by this species in the defense of nest. Cerebral indices of all the analyzed structures suggest that Vanellus c. chilensis is a progresive bird.
\end{abstract}

Keys words: Vanellus c. chilensis, neuroanatomy, relative size, ecology, behavior.

\section{INTRODUCCIÓN}

La gran radiación adaptativa de las aves produjo como resultado profundas modificaciones de sus sistemas corporales y en particular del nervioso. El encéfalo presenta características interesantes, destacándose un marcado desarrollo de los hemisferios cerebrales. Este incremento se refleja en los cuerpos estriados, responsables de los comportamientos instintivos y de los mecanismos relacionados con la memoria y el aprendizaje, constituyendo el centro de coordinación de todo el encéfalo (Pirlot 1989).

Existe una estrecha relación entre el volumen de un determinado componente encefálico y su funcionalidad, determinando que un mayor uso del mismo produce un aumento en el volumen y que se dan interesantes asociaciones con ciertos aspectos bioecológicos (Portmann 1946, 1947, Bang 1960, Cobb 1960, Pearson 1972, Pettigrew \& Konishi 1976, Pirlot \& Jolicouer 1982, Bennet \& Harvey 1985, Bee de Speroni \& Pirlot 1987 , Boire 1989, Pirlot 1989, Alama \& Bee de Speroni 1992, Bee de Speroni \& Carezzano 1992, 1993, 1995, Carezzano \& Bee de Speroni 1995, 1998, Fernández et al. 1997, Núñez \& Bee de Speroni 1997).

Carezzano \& Bee de Speroni $(1995,1998)$ analizaron las variaciones cuantitativas encefálicas en representantes de diversas familias de aves de ambientes acuáticos (Ardeidae, Podicipedidae, Rallidae y Anatidae), determinando similitudes neuroanatómicas en ciertas áreas encefálicas. Estos autores concluyen que tales semejanzas son 
el resultado de un proceso de convergencia evolutiva.

En el presente trabajo se analiza cuantitativamente la organización cerebral en un representante de la familia Charadriidae; Vanellus c. chilensis Molina 1782 (tero o queltehua). Ave ubicua que comparte características de especies acuáticas y terrestres estrictas, y que presenta además ciertos aspectos ecoetológicos muy peculiares como por ejemplo el ser altamente agresiva si su nidada está en peligro; el poseer un variado repertorio de vocalizaciones, tener ritmos de actividad diurnos, ser carnívora, etc. (Palermo 1985).

El objetivo de este estudio es estimar la composición porcentual (indicador funcional) y los índices cerebrales (indicador filogenético) del encéfalo total, siete de sus componentes y los núcleos de relevo de tres vías sensitivas en Vanellus c. chilensis, a fin de establecer relaciones entre el desarrollo de dichas estructuras y su posible asociación con ciertos aspectos bioecológicos de esta ave.

\section{MATERIALES Y MÉTODOS}

Se capturaron en el departamento Juárez Celman, provincia de Córdoba, dos ejemplares machos adultos de Vanellus c. chilensis. Las aves capturadas se anestesiaron con éter sulfúrico y se perfundieron intracardíacamente por el ventrículo izquierdo con formalina (una parte de formol comercial en siete de agua destilada). A cada ejemplar se les determinó la masa corporal $(307,5$ g). Posteriormente se procedió a extraerles los encéfalos para consignar sus masas $(2,55 \mathrm{~g})$. Los valores de la masa corporal y encefálica, son el promedio de los dos ejemplares utilizados.

Los encéfalos se procesaron según la técnica de inclusión en parafina, se cortaron seriadamente en secciones transversales a $10 \mathrm{~mm}$ y se colorearon con Nissl (Cressyl violet). Los cortes se fotografiaron a intervalos regulares y se demarcaron las siguientes áreas neuronales: bulbos olfatorios (BO), estriado propiamente dicho $(\mathrm{St})$, integrado por paleoestriado primitivo y aumentado; arquiestriado (Ast), neoestriado (Nst), integrado por hiperestriado ventral, ectoestriado y neoestriado; Wulst (W), consituido por el hiperestriado dorsal e hiperestriado accesorio; tecto óptico (TO) y cerebelo (Cb). También se delimitaron los siguientes núcleos: ectoestriado (E), núcleo mesencefálico lateral parte dorsal (MLd), núcleo basal prosencefálico (NBP), núcleo sensorial principal del nervio trigémino $(\mathrm{PrV})$, núcleo ovoide $(\mathrm{Ov})$ y núcleo rotundo (incluye núcleo triangular) (Rt). Estas áreas fueron delimitadas utilizando el atlas estereotáxico de Karten \& Hodos (1967).

A fin de obtener el volumen del encéfalo total y de los campos neuronales anteriormente mencionados, los encéfalos se procesaron según el método volumétrico (Stephan et al. 1981).

El cálculo de los índices cerebrales (Portmann 1946, 1947) se basa en la elección arbitraria de una forma aviaria como base de comparación para estimar el desarrollo relativo de un área encefálica dada. Los índices se obtienen de la relación entre la masa de un determinado componente encefálico sobre la cifra basal, la que representa la masa encefálica del tronco cerebral de un galliforme hipotético de la misma masa corporal que la especie en estudio. Para el cálculo de la cifra basal se empleó la ecuación alométrica de Dubois (1897) en la que Portmann basó sus estudios $\mathrm{y}=\mathrm{b} \mathrm{x}^{\mathrm{k}}$, donde y es la masa encefálica, x la masa corporal, $\mathrm{b}$ es un factor específico de encefalización y k es el coeficiente de encefalización.

Portamnn en sus trabajos no menciona el valor de la constante b, Bee de Speroni \& Pirlot (1987) asumieron que trabajó con los valores de Gallus gallus, los cuales son $\mathrm{y}=550 \mathrm{~g} \mathrm{y} \mathrm{x}=0,437 \mathrm{~g}$, con un $\mathrm{k}$ de 0,52 . Con estos valores se obtuvo el valor $\mathrm{de} b=0,016426$.

\section{RESULTADOS}

Los valores porcentuales del encéfalo total y siete de sus componentes de Vanellus c. chilensis, los que se comparan con los de Egretta thula (garza chica), Podiceps rolland (pimpollo o macá) y Fulica armillata (tagua o gallareta) se presentan en la Tabla 1. Las tres últimas especies fueron analizadas por Carezzano \& Bee de Speroni (1995). La Tabla 2 exhibe los porcentajes de los núcleos de relevo de las tres vías sensitivas y la Tabla 3 los índices cerebrales del encéfalo total y los siete componentes estudiados.

Cabe acotar que por razones metodológicas, económicas y ecológicas se analizaron solamente dos ejemplares y se asume por lo tanto que los datos obtenidos carecen de valor estadístico. Sin embargo, según lo señalado por Stephan \& Kuhn (1981), se logra una mejor información si se estudian diversas especies en vez de numerosos ejemplares de la misma.

\section{DISCUSIÓN}

Los resultados obtenidos se discuten empleando el concepto de cociación introducido por Pirlot (1986) como una relación entre características 
TABLA 1

Volumen de los componentes encefálicos expresados en porcentajes en relación al volumen del encáfalo total en Vanellus c. chilensis, Egretta thula, Podiceps rolland y Fulica armillata;

(BO) bulbos olfatorios, (St) estriado propiamente dicho, (Ast) arquiestriado, (Nst) neoestriado, (W) Wulst, (Tel) teléncefalo, (TO) tecto óptico, $(\mathrm{Cb})$ cerebelo

Volume of the component in percentages of the encephalic total volume in Vanellus c. chilensis, Egretta thula, Podiceps rolland and Fulica armillata; (BO) bulbus olfactorius, (St) striatum, (Ast) archistriatum, (Nst) neostriatum, (W) Wulst, (Tel) telencephalon, (TO) tectum opticum, (Cb) cerebellum

\begin{tabular}{|c|c|c|c|c|}
\hline & Vanellus c. chilensis & Egretta thula* & Podiceps rolland* & Fulica armillata* \\
\hline $\mathrm{BO}$ & 0,28 & 0,27 & 0,65 & 0,86 \\
\hline St & 11,89 & 7,65 & 10,58 & 12,70 \\
\hline Ast & 1,58 & 1,21 & 2,72 & 1,85 \\
\hline Nst & 36,64 & 36,75 & 34,14 & 41,36 \\
\hline $\mathrm{W}$ & 11,93 & 5,44 & 5,67 & 7,76 \\
\hline Tel & 68,53 & 54,63 & 57,50 & 68,20 \\
\hline TO & 13,46 & 12,29 & 10,20 & 6,48 \\
\hline $\mathrm{Cb}$ & 11,60 & 16,88 & 14,00 & 9,95 \\
\hline
\end{tabular}

*De Carezzano \& Bee de Speroni (1995)

cuantitativas (correlación) y caracteres cualitativos (asociación). A continuación se describen las estructuras analizadas discutiendo su relevancia.

\section{Telencéfalo}

La topología telencefálica de Vanellus c. chilensis es de tipo dorsofrontal según Stingelin (1958), a semejanza de Egretta thula y Podiceps rolland
(Carezzano \& Bee de Speroni 1995). Esta estructura cerebral es la más voluminosa $(68,53 \%)$.

\section{Bulbos olfatorios}

Los $\mathrm{BO}$ son los órganos principales relacionados con el sentido del olfato. En la mayoría de las aves son considerados componentes en regresión (Bang \& Cobb 1968).

Valores porcentuales relativos de los núcleos de relevo de la vía trigeminal, visual y acústica en Vanellus c. chilensis, Egretta thula, Podiceps rolland y Fulica armillata; (PrV) núcleo sensorial principal del nervio trigémino, (NBP) núcleo basal prosencefálico, (TO) tecto óptico, (Rt) núcleo rotundo, (E) ectoestriado, (MLd) núcleo mesencefálico lateral parte dorsal, (Ov) núcleo ovoide

Percentual relative values of the relevous nuclei of the trigeminal, visual and acoustic pathways in percentages in Vanellus c. chilensis, Egretta thula, Podiceps rolland and Fulica armillata; (PrV) nucleus sensorialis principalis nervi trigemini, (NBP) nucleus basalis prosencephali, (TO) tectum opticum, (Rt) nucleus rotundus, (E) ectostriatum, (MLd) nucleus mesencephalic lateralis pars dorsalis, (Ov) nucleus ovoidalis

\begin{tabular}{lcccc}
\hline & Vanellus c. chilensis & Egretta thula* & Podiceps rolland $^{*}$ & Fulica armillata* $^{*}$ \\
\hline PrV & 0,02 & 0,02 & 0,02 & 0,01 \\
NBP & 0,15 & 0,153 & 0,258 & 0,062 \\
Rt & 0,34 & 0,29 & 0,23 & 0,18 \\
E & 1,21 & 1,18 & 0,99 & 0,95 \\
MLd & 0,09 & 0,05 & 0,07 & 0,10 \\
Ov & 0,04 & 0,02 & 0,02 & 0,04 \\
\hline
\end{tabular}

*De Carezzano \& Bee de Speroni (1995) 


\section{TABLA 3}

Índices cerebrales de Vanellus c. chilensis, Egretta thula, Podiceps rolland y Fulica armillata; (CB) cifra basal, (BO) bulbos olfatorios, (St) estriado propiamente dicho, (Ast) arquiestriado, (Nst) neoestriado, (W) Wulst, (Tel) teléncefalo, (TO) tecto óptico, (Cb) cerebelo

Cerebral indices of Vanellus c. chilensis, Egretta thula, Podiceps rolland and Fulica armillata; (CB) basal number, (BO) bulbus olfactorius, (St) striatum, (Ast) archistriatum, (Nst) neostriatum, (W) Wulst, (Tel) telencephalon, (TO) tectum opticum, $(\mathrm{Cb})$ cerebellum

\begin{tabular}{lcccc}
\hline & Vanellus c. chilensis & Egretta thula $^{*}$ & Podiceps rolland $^{*}$ & Fulica armillata $^{*}$ \\
\hline CB & 0,32 & 0,37 & 0,29 & 0,58 \\
BO & 0,03 & 0,03 & 0,05 & 0,06 \\
St & 1,02 & 0,77 & 0,78 & 0,91 \\
Ast & 0,14 & 0,12 & 0,20 & 0,13 \\
Nst & 3,15 & 3,71 & 2,51 & 2,96 \\
W & 1,03 & 0,55 & 0,42 & 0,56 \\
Tel & 5,23 & 5,52 & 4,23 & 0,89 \\
TO & 1,16 & 1,24 & 0,75 & 0,75 \\
Cb & 1,00 & 1,71 & 1,03 & 0,71 \\
\hline
\end{tabular}

*De Carezzano \& Bee de Speroni (1995)

Cobb (1960) y Bang \& Cobb (1968) citan que las aves acuáticas presentan los bulbos olfatorios muy voluminosos. Algunas especies lo emplean en la búsqueda del alimento (Bang 1960), otras para identificar feromonas en el cortejo (Balthazart \& Schoffeniels 1979) y en la orientación (Benvenuti 1973).

Los BO en Vanellus c. chilensis $(0,28 \%)$ presentan un desarrollo similar al de Egretta thula $(0,27 \%)$ e inferiores a los de Podiceps rolland $(0,65 \%)$ y Fulica armillata $(0,86 \%)$.

El valor estimado en Vanellus c. chilensis está en relación con su dieta carnívora. Es un ave depredadora que utiliza la vista para detectar sus presas, entre las que se incluyen anélidos, insectos (coleópteros, ortópteros y formícidos terrestres y larvas de dípteros acuáticos) que caza caminado en playas con o sin vegetación, aguas someras o incluso en lugares alejados del agua, además pueden llegar a devorar ejemplares juveniles de anfibios y reptiles (Gallardo 1970, Bucher \& Herrera 1981).

\section{Estriado propiamente dicho}

Pearson (1972) menciona que el St se asocia con reflejos condicionados (comportamiento alimenticio, arreglo y lubricación de plumas, etc.). Constituye un sistema de coordinación motriz. Integra patrones de orientación entre los objetos, elaborados a nivel del neoestriado, y el sistema de control de la postura. Estudios fisiológicos reve- lan que el deterioro de esta área provoca apatía, falta de reacción a estímulos y ausencia de conductas de escape, temor, vuelo y ataque (Salzen \& Parker 1975).

El buen desarrollo del estriado en Vanellus $c$. chilensis $(11,89 \%)$ se asocia con lo mencionado en el párrafo anterior, ya que muestra gran precisión en sus vuelos, especialmente los de amenaza, los movimientos desplegados en el cortejo y el tanteo de sus patas, para movilizar a las lombrices que están bajo tierra.

\section{Arquiestriado}

El Ast es una extensa área de integración autónoma con un importante papel en la respuesta al miedo cuya estimulación produce conductas de ataque y fuga (Pearson 1972).

El valor relativo estimado en Vanellus $c$. chilensis $(1,58 \%)$ es superior al de Egretta thula $(1,21 \%)$ e inferior al de Podiceps rolland $(2,72$ $\%)$ y Fulica armillata $(1,85 \%)$.

Vanellus c. chilensis es un ave extremadamente celosa y vigilante, en especial durante la época de nidificación en la que aumenta su irritabilidad. En esta época ataca con furia a toda ave de presa que se acerque al nido, además presenta comportamientos de distracción, tales como el de agacharse en otros lugares fingiendo cubrir la postura o bien simular estar herido. 


\section{Neoestriado}

Integra información que coordina la orientación de los movimientos corporales, particularmente del cuello, pico y miembros hacia objetos u otros estímulos externos (Salzen \& Parker 1975). En la porción ventrolateral se proyecta la vía quinto frontal implicada en el control del picoteo, en la identificación táctil y toma del alimento. En el ectoestriado releva la vía visual tecto fugal, utilizada en mayor proporción por aves de hábitos diurnos. En la porción caudal finaliza la vía auditiva ascendente (Karten 1968).

La cifra obtenida en Vanellus c. chilensis $(36,64$ $\%)$ es similar a la de Egretta thula $(36,75 \%)$ y Podiceps rolland $(34,14 \%)$ e inferior a la de Fullica armillata $(41,36 \%)$. Este valor se asocia con los hábitos diurnos de esta ave, quien localiza visualmente sus presas a las que captura con su fuerte pico. Además posee vocalizaciones inconfundibles y cuando lo hace en forma alternada $y$ sincronizada puede provocar la alarma de otros congéneres.

\section{Wulst o eminencia sagital}

El desarrollo del W está relacionado con la visión binocular y desempeña un papel importante en el aprendizaje, puesto que es asiento de la memoria (Boire 1989). Asimismo se asocia con la habilidad de resolver problemas complejos e inesperados, como la obtención del alimento, lugar de nidificación, defensa de la nidada, etc. (Mazzi \& Fassolo 1977). Este componente se relaciona juntamente con el hiperestriado ventral en la orientación (Salzen \& Parker 1975).

La cifra estimada en Vanellus chilensis $(11,93 \%)$ es muy alta en relación a la de las otras especies (Egretta thula $=5,44 \%$; Podiceps rolland $=5,67 \%$ y Fulica armillata $=7,76 \%$ ), lo que concuerda con los complejos comportamientos defensivos que exhibe Vanellus $c$. chilensis tales como, los vuelos en picada, despliegue de amenaza, de ala caída fingiendo estar herido, despliegue del falso nido, etc. Nidifica en el suelo en forma simple con material vegetal y detritos, seleccionando generalmente sitios de pastizales que permitan el camuflaje de las crías (Palermo 1985).

\section{Tecto óptico}

El sentido más desarrollado en las aves es la vista, con ella detectan el alimento, los predadores, etc. (Pearson 1972). El TO es un centro de relevo de la vía tecto fugal, la cual es empleada principalmente por aves diurnas (Güntürkün 1991).

Vanellus c. chilensis posee un valor relativo de $13,46 \%$, superior al de Egretta thula (12,29\%), Podiceps rolland $(10,20 \%)$ y Fullica armillata $(6,48 \%)$. Esto se relaciona con el hecho de que la visión desempeña un rol importante en la búsqueda del alimento y detección de predadores en Vanellus c. chilensis, quien habita preferentemente campos abiertos que le aseguren una amplia visibilidad, y no se aventura en sitios de vegetación cerrada (Bucher \& Herrera 1981, Palermo 1985).

\section{Cerebelo}

El cerebelo está implicado en el mantenimiento del equilibrio y la integración motriz del organismo, controla la postura y la coordinación de movimientos finos y precisos (Pearson 1972).

El valor relativo exhibido por Vanellus $c$. chilensis $(11,60 \%)$, inferior a los de Egretta thula $(16,88 \%)$ y Podiceps rolland (14,00\%), pero superior al de Fulica armillata $(9,95 \%)$, se asocia con la gran precisión exhibida por Vanellus c. chilensis en sus distintos vuelos como los ya mencionados al discutir los resultados del W.

\section{Vía quinto frontal}

Esta vía está implicada en el control del picoteo (busqueda de alimento), en la toma de partículas alimenticias (Schall \& Deluis 1986) y en la localización táctil de las presas (Gerristen \& Meiboom 1986). El núcleo sensorial principal del nervio trigémino (PrV) recibe la información de los receptores táctiles del pico y de la cavidad oral (Wild et al. 1985). Las eferencias del PrV, que son relevadas en el diencéfalo y el núcleo basal prosencefálico (NBP) constituyen la vía quinto frontal o trigeminal (Wild et al. 1985). Las eferencias del NBP llegan al neoestriado frontal (Schall \& Deluis 1986), y por el tracto frontoarquiestriatal al arquiestriado anterior (Wild et al. 1985) y de éste parten vías motoras que alcanzan diversos componentes mielencefálicos que inervan la musculatura mandibular. Este sistema constituye una interfase sensomotriz que controla los movimientos del pico y cuello (Berkhoudt et al. 1982).

Las cifras estimadas para PrV y NBP en Vanellus c. chilensis son de 0,02 y $0,15 \%$, respectivamente; éstas son similares a las de Egretta thula (PrV $=0,02 \%$ y $\mathrm{NBP}=0,15 \%)$. Estos valores se relacionan con los hábitos alimenticios de 
Vanellus c. chilensis, la cual es una ave carnívora, como ya se citara al discutir los resultados de los BO.

\section{Vía visual}

El aspecto más importante del encéfalo de las aves es el enorme desarrollo del sistema visual. Güntürkün (1991) describe en Columba livia tres vías visuales: la tálamo fugal, primera y segunda tecto fugal. La primera tecto fugal es homóloga a la retino-cólico-pulvinoestriatal de los mamíferos (Karten 1968, Karten \& Hodos 1970). En el presente estudio solo se analizó la primera vía tecto fugal. El Rt es uno de los mayores núcleos del tálamo dorsal que envía eferencias al $\mathrm{E}$ y recibe fibras del TO que proyectan al neoestriado intermedio y caudal (Boire 1989).

Los valores obtenidos para Rt y E en Vanellus c. chilensis $(0,34$ y $1,21 \%$, respectivamente), superiores a los de Egretta thula, (Rt $=0,29 \%$ y $\mathrm{E}=1,18 \%)$, Podiceps rolland $(\mathrm{Rt}=0,23 \%$ y $\mathrm{E}=$ $0,99 \%)$ y Fulica armillata $(\mathrm{Rt}=0,18 \%$ y $\mathrm{E}=0,95$ $\%$ ) concuerdan con el papel que juega la vista en Vanellus c. chilensis, ya que no solo la emplea para detectar a sus presas (insectos, pequeños vertebrados) y sino también a sus predadores (Polyborus plancus, Milvago chimango, Tyto alba, etc.) (Bucher \& Herrera 1981, Palermo 1985).

\section{Vía acústica}

La vía acústica tiene su origen en la cóclea, que envía terminaciones nerviosas por los nervios cocleares al mielencéfalo (Boord 1968). Las eferencias de estos núcleos forman el lemnisco lateral que llega al núcleo mesencefálico lateral parte dorsal (MLd) (Boord 1968, Karten 1974). Las proyecciones del MLd terminan en el núcleo ovoide (Ov) del tálamo, que a su vez proyecta sus axones al campo L de Rose en el telencéfalo (Karten 1968). El MLd y el Ov están implicados en la discriminación y aprendizaje de patrones acústicos complejos, como lo es la vocalización específica de cada especie (Leppelsack \& Vogolt 1976, Langer et al. 1981).

Los valores estimados para los núcleos de esta vía en Vanellus c. chilensis (MLd $=0,09 \%$ y Ov $=0,04 \%$ ) indican un buen desarrollo de estos, lo que se asocia con las fuertes y diversas vocalizaciones que emite, tales como las de alarma hacia sus pichones ante la presencia de predadores. Estudios futuros sobre las vocalizaciones de Vanellus c. chilensis permitirán ampliar la presente discusión.

\section{Índices cerebrales}

La cifra basal indica el peso encefálico de un galliforme hipotético del mismo peso corporal que la especie en estudio. Es decir, que si el encéfalo del ave que se analiza no evolucionó, el peso encefálico será igual a la cifra basal. Los índices cerebrales son indicadores filogenéticos independientes del tamaño corporal de las aves. Estos índices revelan el nivel evolutivo y son comparables entre si (Portman 1947).

La cifra basal estimada para Vanellus c. chilensis es elevada (0,32), superior a la de Podiceps rolland $(0,29)$, pero es inferior a la de Egretta thula $(0,37)$ y Fullica armillata $(0,58)$. El teléncefalo alcanza el nivel evolutivo más alto en Vanellus c. chilensis $(5,23)$ y Egretta thula $(5,52)$, le sigue Fullica armillata $(4,89)$ y Podiceps rolland (4,23). En Vanellus c. chilensis el componente telencefálico que presenta el índice superior es el Nst $(3,15)$ y los índices inferiores corresponden a los BO $(0,03)$.

El St exhibe un valor similar en Vanellus $c$. chilensis $(1,02)$ y Fullica armillata $(0,91)$, mayor a los de las otras dos especies (Egretta thula $=$ $0,77$ y Podiceps rolland $=0,78)$. El W en Vanellus c. chilensis muestra el índice más elevado $(1,03)$, que casi duplica al de las otras tres aves (Fulica armillata $=0,56 ;$ Egretta thula $=0,55$ y Podiceps rolland $=0,42$ ).

En cuanto al TO, los índices obtenidos indican que Vanellus c. chilensis $(1,16)$ y Egretta thula $(1,24)$ alcanzan similar grado de progresión, mayor al de las otras dos especies (Podiceps rolland $=0,75$ y Fulica armillata $=0,75)$. El Cb de Vanellus c. chilensis $(1,00)$ exhibe valores semejantes a los de Podiceps rolland (1,03), superior al de Fulica armillata $(0,71)$, e inferior a de Egretta thula $(1,71)$.

En base a los valores obtenidos en todas las estructuras encefálicas analizadas se puede inferir que Vanellus c. chilensis es un ave progresiva (Bee de Speroni \& Pirlot 1987, Fernández et al. 1997).

\section{CONCLUSIONES}

Como resultado del presente estudio se concluye que el Tel es el componente de mayor volumen relativo, siendo el Nst la estructura telencefálica de tamaño superior. El desarrollo de los BO, St , TO, y los núcleos de relevo de las vías visual y trigeminal concuerdan con la dieta carnívora de Vanellus c. chilensis. El tamaño relativo del W y de los núcleos de la vía acústica se asocian con las complicadas tácticas que utiliza esta especie en la 
defensa del nido, como así también con el amplio repertorio de vocalizaciones. Los índices cerebrales de todas las estructuras encefálicas analizadas indican que Vanellus c. chilensis es un ave progresiva.

\section{LITERATURA CITADA}

ALAMA S \& N BEE-DE-SPERONI (1992) Índices cerebrales y composición cuantitativa encefálica en Athene cunicularia y Tyto alba (Strigiformes: Strigidae y Tytonidae). Facena 9: 19-37.

BALTHAZART J \& E SOFFENIELS (1979) Pheromones are involved in the control of sexual behaviour in birds. Naturwissenschaften 66: 55-56.

BANG BG (1960) Anatomical evidence for olfactory function in some species of birds. Nature 188: 547549.

BANG BG \& S COBB (1968) The size of the olfactory bulb in 108 species of birds. The Auk 85: 55-61.

BEE-DE-SPERONI N \& F CAREZZANO (1992) Composición cuantitativa encefálica en Polyborus $c h$. chimango Vieillot, desde una perspectiva ecoetológica (Falconidae). Revista de la Asociación de Ciencias Naturales del Litoral (Argentina) 23: 1-8.

BEE-DE-SPERONI N \& F CAREZZANO (1993) Estudio cuantitativo de componentes encefálicos en tres especies de tinámidos, con un enfoque ecoetológico (Aves: Tinamidae). Revista de la Facultad de Ciencias Médicas de Córdoba (Argentina) 51: 5-8.

BEE-DE-SPERONI N \& F CAREZZANO (1995) Volumetric analysis of the visual, trigeminal and acoustic nuclei in three bird families (Rheidae, Tinamidae, Spheniscidae). Marine Ornithology 23: 11-15.

BEE-DE-SPERONI N \& P PIRLOT (1987) Relative size of avian brain components of the Magellan penguin, the greater rhea and the tataupa tinamou. Cormorant 15: 7-22.

BENNETT PM \& PH HARVEY (1985) Relative brain size and ecology in birds. Journal of Zoology 207: 151169.

BENVENUTI S (1973) Homing perfomances of inexperienced and directionally trained pigeons subjected to olfactory nerve section. Journal of Comparative Physiology A 83: 83-91.

BERKHOUDT H, BG KLEIN \& HP ZEIGLER (1982) Afferents to the trigeminal and facial motor nuclei in pigeon (Columba livia L.): central connections of jaw motoneurons. Journal Comparative of Neurology 209: 301-312.

BOIRE D (1989) Comparaison de l'encephale, de ses subdivisions et de relais visuels, trijemeaux et acoustiques chez 28 espèces d'oiseaux. Tesis Doctoral, Universidad de Montreal, Montreal, Canada. 435 pp.

BOORD RL (1968) Ascending projections of primary cochlear nuclei and nucleus laminaris in pigeon. Journal Comparative of Neurology 133: 535-542.

BUCHER H \& G HERRERA (1981) Comunidades de aves acuáticas de la laguna Mar Chiquita (Córdoba, Argentina). Ecosur (Argentina) 8: 91-120.
CAREZZANO F \& N BEE-DE-SPERONI (1995) Composición volumétrica encefálica e índices cerebrales en tres aves de ambiente acuático (Ardeidae, Podicipedide, Rallidae). Facena 11: 75-83.

CAREZZANO F \& N BEE-DE-SPERONI (1998) Análisis cuantitativo encefálico de Anas versicolor Vieillot 1816 (Aves: Anatidae). Natura Neotropicalis 29: 915 .

COBB S (1960) Observations on the comparative anatomy of the avian brain. Perspective in Biology and Medicine 3: 383-408.

DUBOIS E (1897) Sur le rapport du poids de l'encéphale avec la grandeur du corps chez les Mammifères. Bulletin of the Society Anthropology of Paris 4: 337376.

FERNÁNDEZ P, F CAREZZANO \& N BEE-DE-SPERONI (1997) Análisis cuantitativo encefálico e índices cerebrales en Aratinga acuticaudata y Myiopsitta monachus de Argentina (Aves: Psitaccidae). Revista Chilena de Historia Natural 70: 269-275.

GALLARDO JM (1970) Estudio ecológico sobre los anfibios y reptiles del sudoeste de la Provincia de Buenos Aires, Argentina. Revista del Museo Argentino de Ciencias Naturales de Zoología (Argentina) 10: 2763.

GERRISTEN AFC \& A MEIBOOM (1986) The role of touch in prey density estimation by Calidris alba. Netherlands Journal of Zoology 36: 530-562.

GÜNTÜRKÜN O (1991) The functional organization of the avian visual system. En: Andrew RJ (ed) Neural and behavioural plasticity: the use of the domestic chick as a model: 92-105. Oxford University Press, Oxford, United Kingdom.

KARTEN HJ (1968) The ascending auditory pathways in the pigeon (Columbia livia). II. Telencephalic projections of the nucleus ovoidalis thalami. Brain Research 11: 134-153.

KARTEN HJ (1974) The structural organization of the avian brain: an overview. En: Goodman IJ \& M Schein (eds) Birds, brain and behavior: 29-73. Academic Press, New York, New York.

KARTEN HJ \& W HODOS (1967) A stereotaxic atlas of the brain of the pigeon (Columba livia). The John Hopkins Press, Baltimore, Maryland. i-ix +200 pp.

KARTEN HJ \& W HODOS (1970) Telencephalic projections of the nucleus rotundus in the pigeon (Columba livia). Journal Comparative of Neurology 150: 253-278.

LANGER G, D BOMKE \& H SCHEICH (1981) Neuronal discrimination of natural and synthetic vowels in the field L of trained Mynard birds. Experimental Brain Research 13: 11-24.

LEPPELSACK HJ\& M VOGOLT (1976) Responses of auditory neurons in the forebrain of a songbird during stimulation with species-specific sounds. Journal Comparative of Physiology 107: 263-274.

MAZZI V \& A FASSOLO (1977) Introduzione alla neurologia comparata dei vertebrati. Boringhieri, Torino, Italy. $621 \mathrm{pp}$

NÚÑEZ HA \& N BEE-DE-SPERONI (1997) Análisis cuantitativo del desarrollo encefálico prenatal en la vizcacha de las pampas Lagostomus maximus maximus (Rodentia: Chinchillidae), Argentina. Revista Chilena de Historia Natural 70: 59-65. 
PALERMO A (1985) El tero. Centro Editor de América Latina S.A., Buenos Aires, Argentina. 32 pp.

PEARSON R (1972) The avian brain. Academic Press, New York, New York. 658 pp.

PETTIGREW JD \& M KONISHI (1976) Monocular deprivation in kittens impairs the spatial resolution of geniculate neurones. Nature 24: 753-754.

PIRLOT P (1986) Understanding taxa by comparing brain. Perspectives in Biology and Medicine 29: 499-509.

PIRLOT P (1989) Brains and behaviours. Orbis, Québec, Canada. 360 pp.

PIRLOT P \& P JOLICOUER (1982) Correlations between major brain regions in Chiroptera. Brain Behaviour and Evolution 20: 172-181.

PORTMANN A (1946) Etudes sur la cérébralisation chez les oiseaux. I. Alauda 14: 2-20.

PORTMANN A (1947) Etudes sur la cérébralisation chez les oiseaux. II. Alauda 15: 1-15.

SALZEN E \& DM PARKER (1975) Arousal and orientation functions of the avian telencephalon. En: Wright PG \& DM Vowles (eds) Neural and endocrine aspects of behavior in birds: 205-241. Elsevier, Amsterdan, The Netherlands.
SCHALL U \& JD DELUIS (1986) Sensory inputs to the nucleus basalis prosencephali, a feeding-pecking center in the pigeon. Journal Comparative of Physiology A 159: 33-41.

STEPHAN H, H FRAHM \& G BARON (1981) New and revised data on volumes of brain structures in insectivores and primates. Folia Primatologica 35: 129.

STEPHAN H \& HJ KUHN (1981) The brain of Micropotamogale lamote $i$ Heim de Balsac. Zeitschrift für Säugetierkunde 47: 129-142.

STINGELIN W (1958) Vergleichend Morphologische Undersuchungen am Vorderhirn der Vögel auf Cytologischer and Cytoarchitectonischer Grundlage. Helbing \& Lichtenhahn. Basil, Switzerland. 123 pp.

WILD JM, JA ARENDS \& HP ZEIGLER (1985) Telencephalic connections of the trigeminal system in the pigeon (Columba livia). Journal Comparative of Neurology 234: 441-464. 